\title{
Measuring Force Intensity and Direction with a Spatially Resolved Soft Sensor for Biomechanics and Robotic Haptic Capability
}

\author{
Artémis Llamosi, ${ }^{1,2, *}$ and Séverine Toussaint ${ }^{2,3}$
}

\begin{abstract}
Possessing a sense of touch is fundamental for robots to operate outside controlled environments. Nevertheless, pressure and force-sensing technologies are still less mature than vision or proprioception solutions in commercial robots. In this study we present a novel spatially resolved force sensor that allows dynamic measurement of both the intensity and the direction of forces exerted on a custom-shaped surface. Originally designed for biomechanics of arboreal primates, this sensor meets several challenges in engineering robotic skin. Of importance, its ability to measure tangential forces would be instrumental for robotic hands to grasp deformable and unknown objects. Based on optical measurements of deformations, this array sensor presents a soft, biocompatible, weather resistant body, immune to electromagnetic interferences. Central to the costeffectiveness of this solution is an architecture where a single image sensor handles hundreds of force measurement points simultaneously. We demonstrate the performance of this sensor in reconstructing normal and slantwise forces on a flat prototype adapted to forces under 3 N. Finally, we discuss the broad range of possible customizations and extensions for applications in biomechanics and robotics.
\end{abstract}

Keywords: biomechanics, robotic skin, sensor array, 3D force sensor, robot haptics

\section{Introduction}

A DDING SENSES TO ROBOTS allows them to serve outside controlled environments by apprehending autonomously novel situations and objects. Sensing robots can therefore undertake a wider and more general array of tasks. Although they are still under very active development, vision and proprioception/kinesthesis are now standard features of commercial robots. In comparison, arrays of tactile sensors for robots (i.e., robotic skin) are more an active area of research than a mature technology portfolio.

Yet, touch and proprioception work together in forming what is often called somesthesis and is the basis for haptic capabilities, that is, exploring the environment by interacting with it. ${ }^{1}$ From a biological perspective, somesthesis is a fundamental sense shared by all animals and is possibly the most ancient sense. ${ }^{2,3}$ We therefore should expect that adding the sense of touch, by enabling complete somesthesis, will improve considerably robots' capacity to safely and autonomously mingle in unknown environments.

There are many engineering challenges in designing a robotic skin including: sensitivity, resistance to wear, geometric adaptability, power consumption, integration, and obviously cost. ${ }^{4}$ In this study, we designed a local directional force-sensing technology that has many interesting properties regarding these aspects. In the spectrum of force-sensing technologies, it can be seen as an array of "stiff sensing pixels" that is an underexplored direction to cope with the

\footnotetext{
${ }^{1}$ Laboratoire Matière et Systèmes Complexes, UMR 7057, Université Paris Diderot \& CNRS, Paris, France.

${ }^{2}$ CRI, Département Frontières du vivant et de l'apprendre, IIFR, Université Paris Descartes, Paris, France.

${ }^{3}$ UFR Sciences Du Vivant, Université Paris Diderot-Paris 7, Sorbonne Universités, Centre de Recherche en Paléontologie-Paris (CR2P, UMR 7207), CNRS/MNHN/UPMC, Paris, France.

*Current address: DREEM S.A.S., Paris, France.

(C) Artémis Llamosi and Séverine Toussaint 2019; Published by Mary Ann Liebert, Inc. This Open Access article is distributed under the terms of the Creative Commons Attribution Noncommercial License (http://creativecommons.org/licenses/by-nc/4.0/) which permits any noncommercial use, distribution, and reproduction in any medium, provided the original author(s) and the source are cited.
} 
typical challenge of decoupling force-induced signals from the deformation or geometry of the substrate. ${ }^{5}$

Unlike most sensors, our soft-sensing array relies on optical measurements. This technical direction, mostly explored in the 2000s usually yielded large, bulky, and highly consuming systems. ${ }^{6}$ Yet, optical systems like the one presented here possess several intrinsic advantages such as an incomparable immunity to electromagnetic interference. In addition, some optical systems ensure an easier integration by separating force measurement location from optical measurement and the possibility to easily adapt the sensitivity and cost of the system by only changing the optical measurement component. Integration advantages of optical system have even motivated converting electrical sensors' output from microelectromechanical systems to light signals. ${ }^{7}$ Moreover, advances in several technical domains such as light-emitting diodes (LEDs), plastic optical fibers (POFs), complementary metal-oxide-semiconductor active image sensor (CMOS) image sensors, and microfabrication help reducing many limitations of previous optical-based sensors.

An efficient robotic skin would probably features a mixture of sensing elements ${ }^{4}$ similar to the human body where sensing units with distinct properties are present in different proportions and densities depending on the body location. ${ }^{8}$ Except a few noticeable exceptions, ${ }^{9-12}$ existing touch sensors are all pressure or contact sensors. ${ }^{13}$ Although pressure or contact information can be sufficient for a wide array of functions, when it comes to grasping, having access to both normal and tangential components of contact force is fundamental, notably for deformable objects, as has been demonstrated on several robotic hands. ${ }^{14-16}$ This is because grasping relies heavily on friction forces and pressure information is insufficient to capture it. Moreover, forces perception has been proven to prevail over geometric consideration in human haptic exploration. ${ }^{17}$ Practically speaking, with pressure information only, it is in general not possible to distinguish whether an object is slipping or not. It would require previous knowledge of the objects' geometry and the interface friction coefficients. Said otherwise, a robotic hand lacking tangential force sensitivity is only capable of dealing with well-known objects.
This project began with the study of animal biomechanics. The technology described in this article was originally developed for measuring small arboreal primate grasping during climbing where friction aspects are crucial: living in arboreal environments requires hands and feet to develop tangential forces while grasping branches to stabilize the body. Measuring dynamically the repartition of contact forces during grasping would be instrumental for biomechanics. To this end, our sensor must to be made in a soft, weather-resistant, and biocompatible material (some animals tend to chew new objects). In addition, the sensor shape (i.e., the sensitive surface geometry) had be adaptable, for example, a cylindrical shape mimicking a branch.

The results here are based on a flat sensor designed for validation and characterization purposes. Given the species we want to study at first (small mammals of $0.5-2 \mathrm{~kg}$ with hands and feet of $2-10 \mathrm{~cm}$ ), the required spatial resolution was of four measurement points per square centimeter, with each measurement point (that we call "cell") having a measurement range of 0 $3 \mathrm{~N}$. Moreover, measurement frequency had to be $30 \mathrm{~Hz}$ at least and the system should be autonomous for field studies.

Serendipitously, characterizing animal grasp entails many similar challenges with the design of robotic grasping hands. We believe this work to be of equal interest to the robotics and biomechanics communities. Many design choices related to animal biomechanics, such as having soft and sensitive elements, are also well suited for robots interacting with humans. Yet, whenever priorities or constrains may differ, we will discuss variants of interest to robotics, leveraging upon the large adaptability of the presented technology.

\section{Materials and Methods}

\section{System overview}

Our sensor is composed of an array of sensing units (cells) made of a soft and elastic material (Fig. 1A). When a contact force is applied, each cell is deformed depending on the local force. Measuring the cell's deformation gives access to the applied force. To measure each cell's deformation, we track the position of a tracer embedded in the elastic material using an optical principle analogous to performing triangulation:

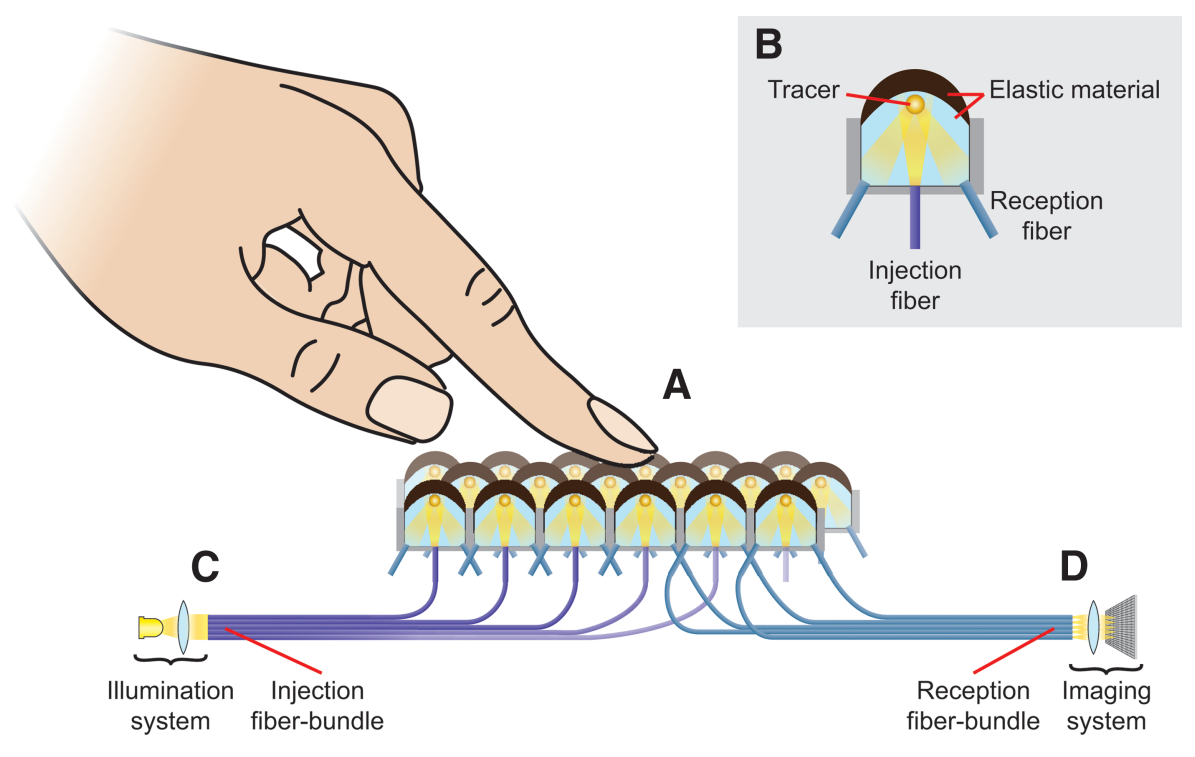

FIG. 1. Overview of the sensor. (A) Array of force-sensing cells. (B) Close-up of a cell. (C) Light injection system and injection optical fiber bundle. (D) Imaging system and reception optical fiber bundle. 
(1) for each cell, a source of light illuminates the tracer, (2) light is reflected and diffused by the tracer, and (3) is collected in three points (Fig. 1B). The intensity of light received in each point is related to the tracer position, and this relation is used to reconstruct the tracer displacement.

As we have a possibly large array of cells, we used several optical fibers to both inject light in each cell and collect light in three points per cell. A single light source illuminates all injection optical fibers (Fig. 1C). All reception optical fibers are imaged at the same time by a single CMOS sensor (Fig. 1D). From acquired images, a simple image processing pipeline extracts single-fiber light-intensity measurements for each frame. Using a mechanistic model of a cell's physics, singlefiber intensity measurements allow to reconstruct tracer displacements in each cell and ultimately, the applied force.

\section{Components description}

The sensor contact surface consists in a tiling of soft, deformable cells of $4 \mathrm{~mm}$ in diameter in our prototype (Fig. 2). Cell design will determine many properties of the final system such as contact quality, measurement range, and precision. The emerging part of cells is approximately semispherical to convert oblique contact forces as a slantwise compression of the cell rather than as a surface shearing force. This is desirable because the latter deformation mode depends more importantly on friction parameters of the interface with object in contact, which are usually unknown. Note that this is particularly relevant for measuring contact force with objects that are themselves deformable or soft to some extent, such as animal hand and feet in our case. Similarly, such dome-shaped cells would be of interest if the sensor surface is itself deformable (e.g., sensors used on a robot hand).

To ensure a simple analytical relation between the exerted force and the cell deformation, we used PDMS (polydimethylsiloxane, Sylgard 184; Dow Corning), an elastomer that follows Hooke's law until $40 \%$ compression. ${ }^{18}$ Given that PDMS is incompressible, the inner part is not deformed by forces applied on the emerged part. PDMS is made from a heat-cured two-component mix. Its mechanical characteristics can be tuned by varying the mix ratio and curing tem- perature. $^{18,19}$ Stiffer PDMS means a larger measurement range as more force can be applied before overshooting the $40 \%$ deformation limit of elasticity. Nevertheless, a stiffer material also means a decreased precision as deformation is smaller for a same force (and therefore harder to detect). Results reported throughout the article have been obtained with a PDMS mix ratio of $1: 15$ and a $24 \mathrm{~h} 40^{\circ} \mathrm{C}$ heat cure.

Cell material needs to be transparent and isolated optically from the environment. In our prototypes, transparent cells were fabricated with a two-step molding procedure allowing to have an outer coating with pigmented opaque PDMS. Within the elastomer, a reflective bead $(\sim 0.5 \mathrm{~mm}$ in diameter) is embedded as a tracer. Cells are embedded in a rigid structure. For our prototypes, the structure was made by precision three-dimensional (3D) printing (Polyjet HD; Stratasys) already including drillings for guiding fibers. Cells were made in hexagonal shape to maximize the sensing surface relative to the residual structure.

POFs are used to transmit light to each cell and to collect it. In addition to being cheaper than silica optical fibers, POF are also mechanically more resistant and bendable. Given that our measurements rely on light intensity only, light pipes can be used instead of POF. For our prototypes, we used multimode, step index POF of $500 \mu \mathrm{m}$ diameter (Super Eska; Mitsubishi Rayon Co. Ltd.). A single collimated light source (35 lm white commercial grade LED) irradiates the injection POF bundle (Figs. 1C and 2). A bundle is a compact arrangement of parallel fibers, embedded in optical epoxy resin, and polished. For each cell, the injection fiber shines light on the tracer and three reception fibers collect the light reflected by the tracer (Fig. 1B). All reception fibers are regrouped in a bundle to be imaged all together (Fig. 1D). The bundle is imaged using a simple objective conjugating the fibers bundle and a CMOS sensor. In our prototypes, we used a 12 bit, global shutter, monochrome USB3.0 camera (IDS camera harboring the e2v Sapphire 1.3M pixel CMOS, EV76C560; Fig. 2).

\section{Data processing and force reconstruction}

At each time frame, an image is taken. Given the magnification of our imaging system, each fiber's image is an $\sim 42$
FIG. 2. Prototype used in this study. Several cell matrices were produced to illustrate various fabrication steps and at the center is an enlarged and uncoated cell.

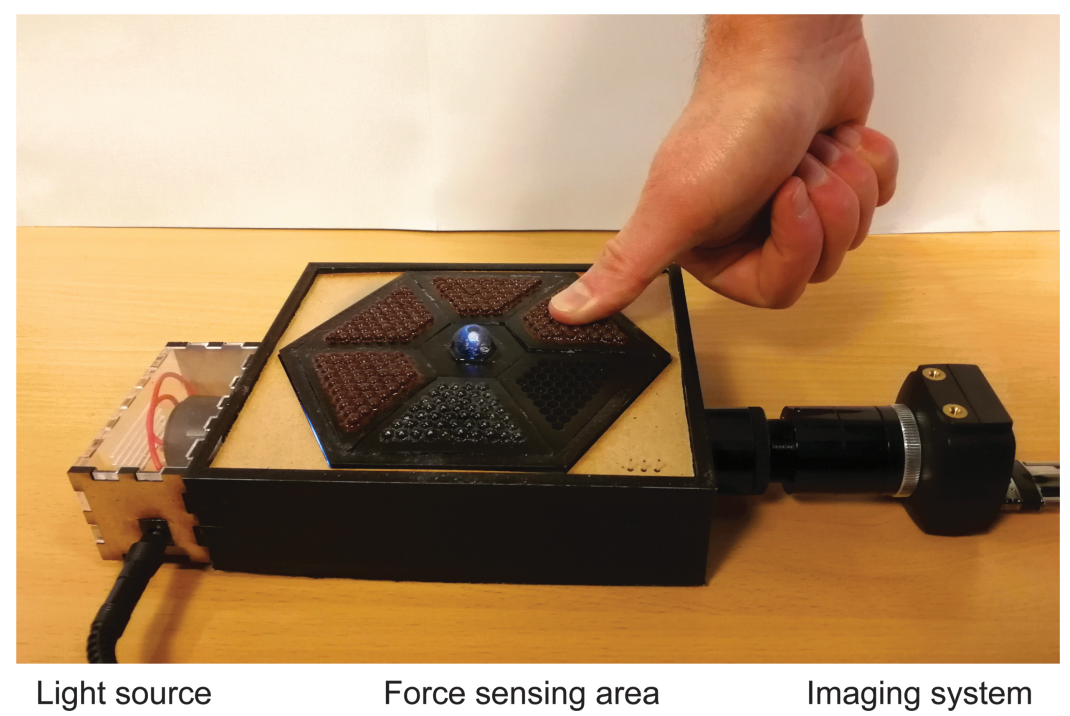

Light source
Imaging system 
A
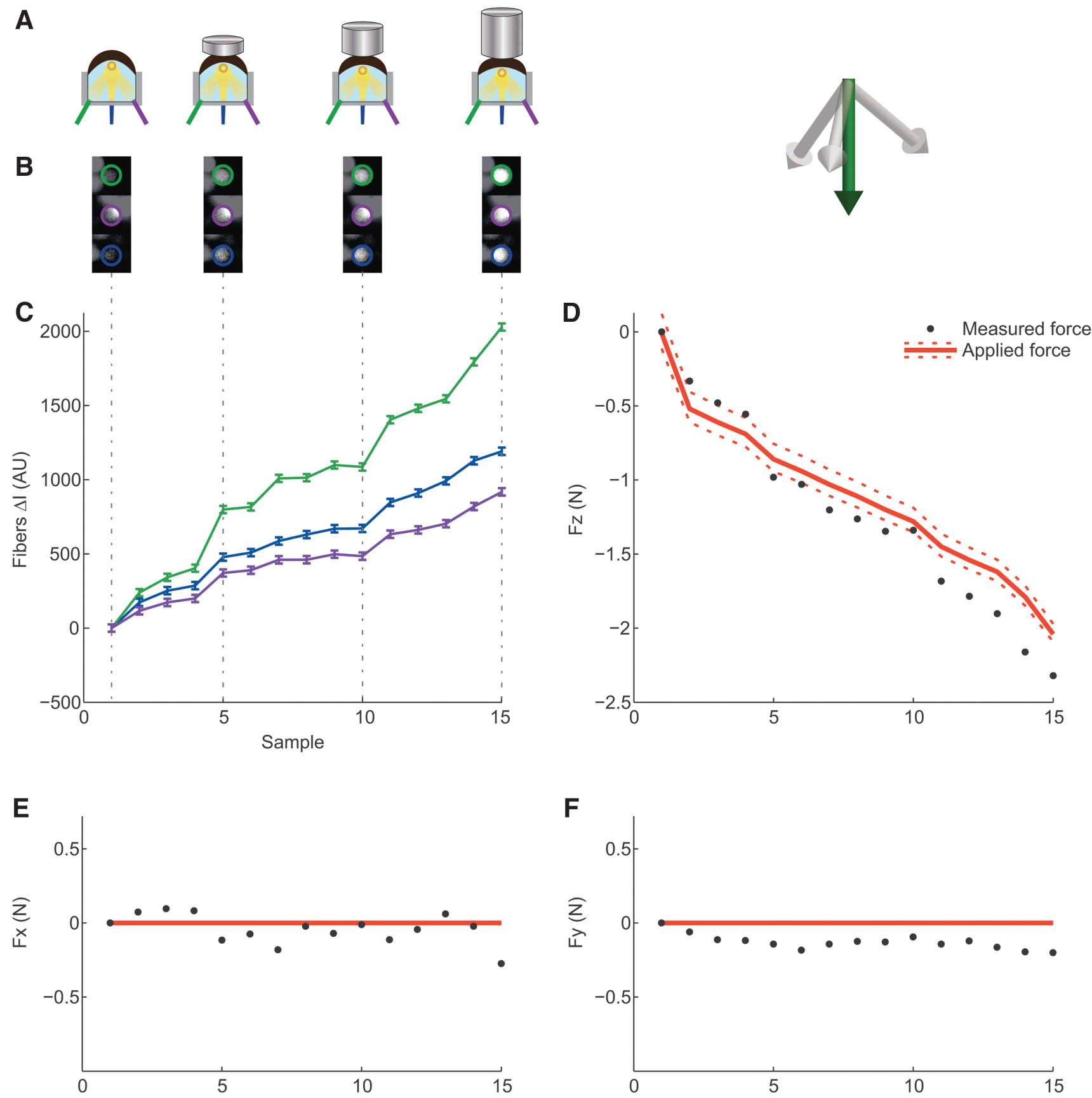

FIG. 3. Reconstruction of a normal indentation force. (A) Sketch view of the applied force and geometric convention. (B) Snapshots of the bundle, centered on the three fibers of the cell of interest, at different time points. (C) Fibers normalized change in intensity $\left(\Delta f_{1}^{t}, \Delta f_{2}^{t}, \Delta f_{3}^{t}\right)_{t=0 \ldots N}$. extracted from bundle images. Error bars represent intensity measurement error (SD). For (A-C), colors distinguish the three different reception fibers. (D) Vertical component of force $\left(F_{z}\right)$ applied (solid red line) and measured (dots). Dotted red line indicates the uncertainty on the applied force. (E, F). Tangential components of force $\left(F_{x}, F_{y}\right)$ applied (solid red line) and measured (dots). For these components, we could not estimate uncertainties in applied force. SD, standard deviation.

pixel diameter disk for $500 \mu \mathrm{m}$ diameter fibers (Fig. 3B). For each frame and each fiber, the average pixel intensity (on a fixed size 28 pixel diameter circle, centered on the fiber's image) is extracted. We note $f_{i}^{t}$ the measured intensity of fiber $i$ taken at frame $t$. For usage in the field, we apply as a preprocessing step an automatic image registering to compen- sate for eventual vibrations causing small displacements of the whole bundle between frames.

We then apply the following two-step normalization and de-noising process: (1) removal of light injection fluctuations (general intensity change) and (2) normalizing relative to absence of force. Using a reference frame taken 

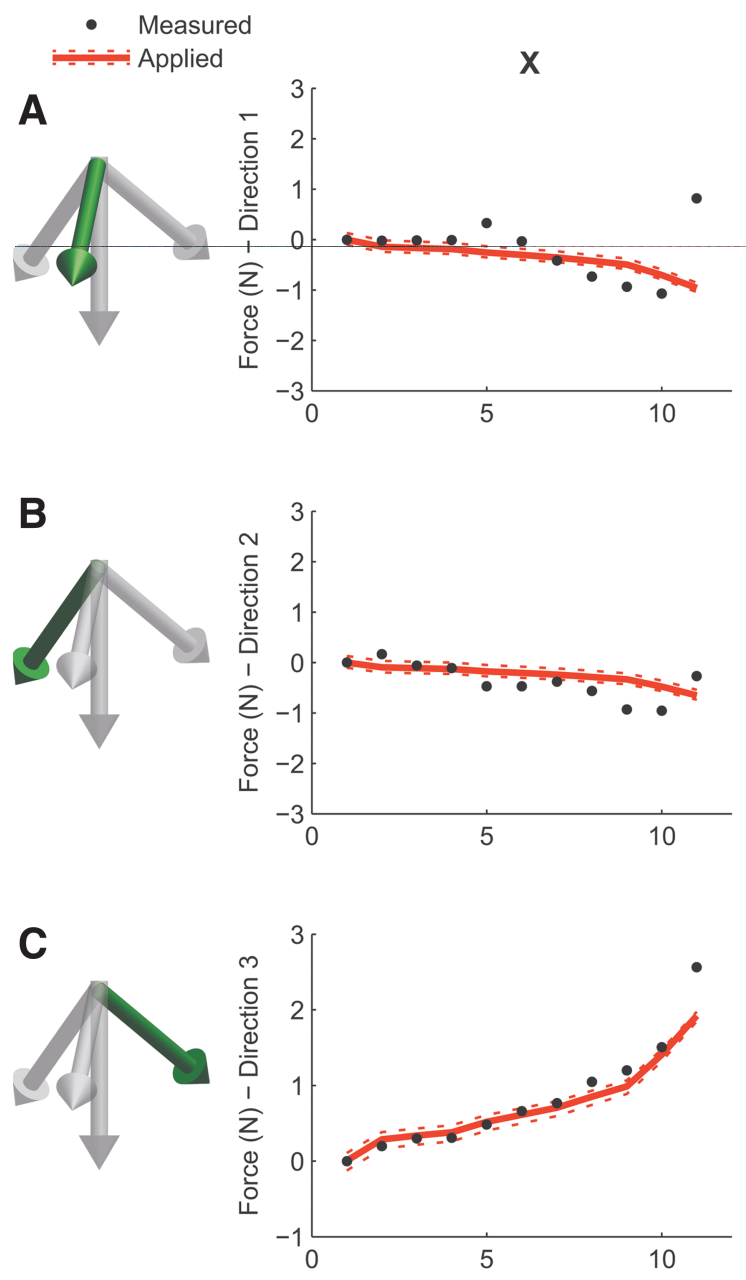
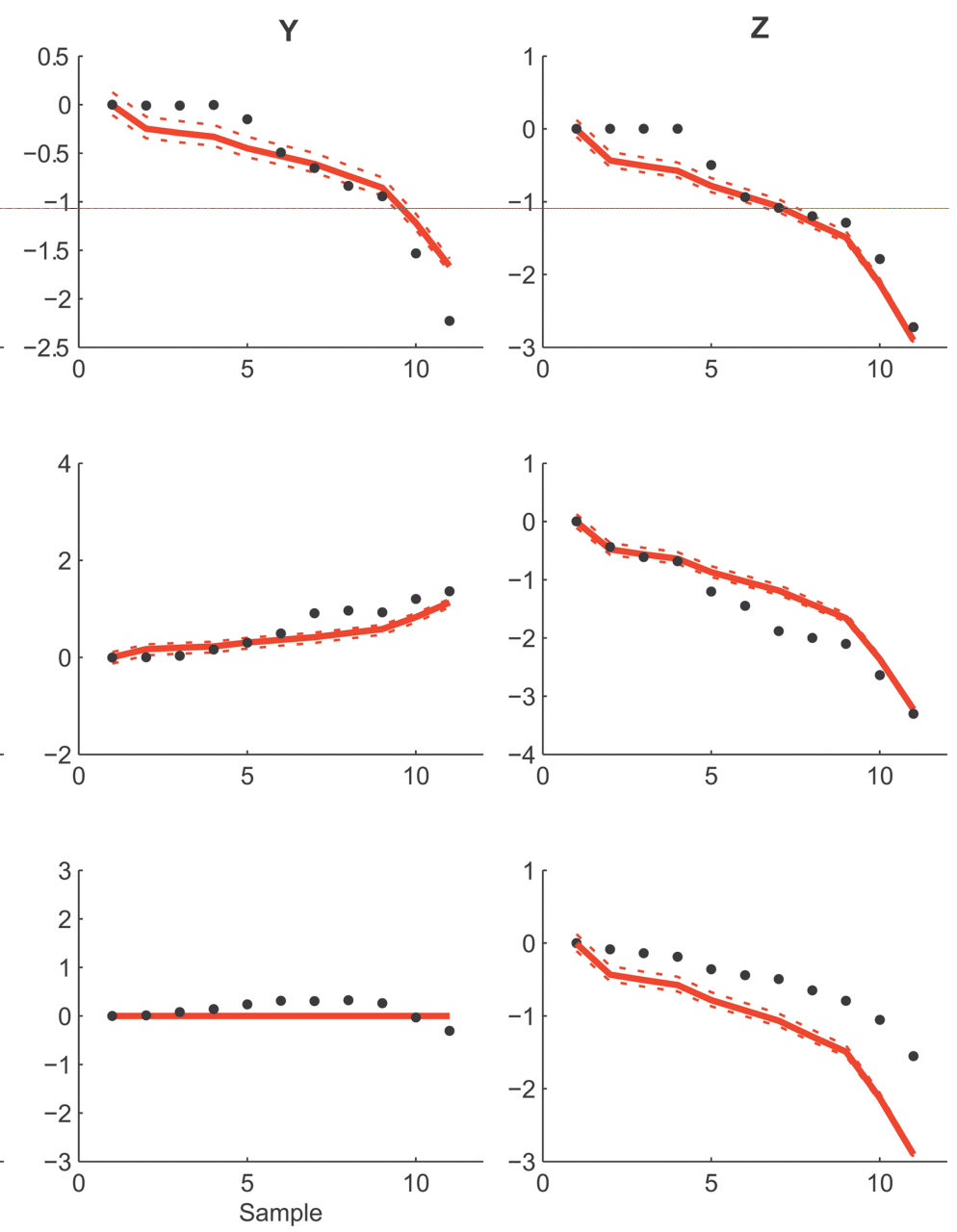

FIG. 4. Reconstruction of slantwise forces. For (A-C), left panes represent $F_{x}$, middle panes, $F_{y}$, and right panes $F_{z}$. For directions 1 and 3 the angle of the applied force to the normal was $22^{\circ}$, whereas for direction 2 , it was $33.5^{\circ}$.

without any force $f_{i}^{0}$ we compute the relative common change in intensity $c c^{t}=\left\langle f_{i}^{t} / f_{i}^{0}\right\rangle_{i}$ and assume such variation is because of global change in injection light intensity at time $t$ relative to the reference frame. Note that a direct measurement of injection light fluctuations allows to obtain a similar correction factor more directly and more reliably. Finally, (1) and (2) yields $\Delta f_{i}^{t}=f_{i}^{t} / c c^{t}-f_{i}^{0}$ which we use in our force reconstruction model (see Fig. 3C for an example of $\Delta f_{i}^{t}$ ).

Single fiber intensity data are injected in a simple model of the physics of a cell. This model integrates:

(1) The geometry of any specific cell (optical fibers orientations and positions, tracer position).

(2) A linear mechanical model of the cell deformation and associated tracer displacement validity of which is restricted to the elastic portion of the deformation. In addition to cell geometry, this includes the compression Young modulus of the elastomer.

(3) A simple light irradiance model capturing the relation between the tracer position and changes in reception fiber's irradiance related to $\Delta f_{i}^{t}$.

This model can be mathematically described as a mapping $F 2 I \underline{\underline{\operatorname{def}}} \vec{F}_{i}^{t} \rightarrow\left(\Delta f_{i 1}^{t}, \Delta f_{i 2}^{t}, \Delta f_{i 3}^{t}\right)$, where $\vec{F}_{i}^{t}$ is the local force vector for cell $i$ at time $t$, and $\Delta f_{i}^{t}$ is the normalized-intensity data from the three fibers of cell $i$. To reconstruct applied forces (Figs. 3D-F. and 4) from intensity measurement, we solve:

$$
\vec{F}_{i}^{* t}=\underset{F}{\operatorname{argmin}}\left\|F 2 I(F)-\left(\Delta f_{i 1}^{t}, \Delta f_{i 2}^{t}, \Delta f_{i 3}^{t}\right)^{2}\right\| .
$$

The force reconstruction process requires parameters that are estimated using a simple calibration procedure. These parameters are as follows: (1) the correspondence between each fiber in the bundle image and the associated specific reception point in a specific cell; (2) a normalization vector for each reception fiber that compensates for both manufacturing variability on reception fiber orientations, and eventual differences in injection and reception fibers transmittance (because of variation in length or fiber-surface quality); (3) the real position of the tracer in each cell to account for manufacturing variability.

Calibration is automated with a custom machine where low-friction pneumatic cylinders apply known forces to each cell (we used M9 Airpel ${ }^{\circledR}$ Anti-Stiction Air Cylinders from Airpot Corp.). For this study, we applied to each cell 18 
different vertical forces and $14 \times 3$ different slantwise forces (along 3 different directions). The reason how reducing the amount of calibration points affects calibration quality has not been investigated yet.

\section{Results}

\section{Force reconstruction}

In a first experiment, increasing normal downward forces (i.e., along the $z$ axis only) were applied on a cell by an air cylinder, yielding 15 points ranging from 0 to $2 \mathrm{~N}$ as given in Figure 3A. From bundle images (Fig. 3B), the variation in fiber intensity, normalized as described previously were extracted. In Figure 3C, we notice that applying force increases the fiber intensity of all fibers. This is expected as the length of the light path going from the emitting fiber to the receiving fiber (through the tracer) decreases with the deformation of the cell cap. If the system was perfect, we would have the same light intensity value along the path for all fibers. In practice, however, some parameters differ between fibers such as fiber transmittance or orientation. Calibration compensates for these fiber-specific variations. From fiber intensities, cell's deformation and the applied force $\vec{F}(t)=\left\{F_{x}(t), F_{y}(t), F_{z}(t)\right\}$ are reconstructed as given in Figure 3D-F. Other experiments (not shown) indicate that our system reconstructs accurately the normal force component until $3 \mathrm{~N}$. Yet, above $2.5 \mathrm{~N}$, tangential components show progressively significant deviations from their expected values. This can happen when the deformation exceeds Hook's law validity domain for the material or as a result of slight misalignments in our force application system. Nevertheless, the system's performance degrades gracefully when compression exceeds the linear assumption threshold and the model becomes more and more inaccurate. In addition, PDMS can endure much higher strains before breaking or being damaged. $^{18}$

In a second experiment, we applied slantwise forces instead of normal forces (Fig. 4). These results demonstrate that our system can reconstruct not only the intensity but also the direction of the applied force.

\section{Measurement precision and key performance factors}

Several parameters of the system impose tradeoffs between precision, cost, speed, and measurement range. Cell material mechanical properties impose the range of measurable forces as the region where deformation is reversible (and ideally linear to ensure easier force reconstruction). Therefore, the higher the compression modulus, the larger is the force measurement range. Material stiffness also plays a role in the force measurement precision: for a given force, a stiffer material will deform less, generating a smaller (and harder to detect) tracer displacement. Therefore, a stiffer material increases the measurement range at the expense of the measurement precision. Accuracy in tracer displacement measurement is driven by both optical and geometric considerations. Cell size and optical isolation, tracer size, optical fiber sizes, and orientation are the most important parameters. Interestingly, for a given combination of the aforementioned parameters, there is a large margin of improvement in choosing the light source and imaging system. The quality of the CMOS/charged coupled device sensor and more generally of the reception bundle imaging system conditions importantly the overall measurement precision and acquisition speed. This large contribution of standard components' quality (such as CMOS sensors) to our systems' performance makes it very versatile and costeffective as will be discussed later.

To summarize, the uncertainty in the measured force comes from the uncertainty in tracer displacement and depends on the material stiffness. Estimating the tracer displacement depends mostly on the precision of fiber intensity measurement. Errors in intensity measurement come from uncorrected fluctuations from the light source, cross-contamination of fiber measurement by halo effect, and CMOS errors in intensity measurements. In our image acquisition conditions, the average standard deviation in fiber intensity was empirically measured at $1.6 \%$ before normalizing (on $>100$ fibers from $>40$ cells and compiling $>10,000$ measurements per fiber across the whole range of forces used in the article) and at $0.59 \%$ after normalizing, which corresponds to a signal-to-noise ratio of 8.31 and 14.0, respectively.

To evaluate more empirically our sensor precision, we conducted a reproducibility experiment where a same force was applied several times with our piston machine. We repeated 10 times 35 different force measurements ranging from 0.5 to $2.5 \mathrm{~N}$ along 4 different force directions. As given in Figure 5, this leads to overall reproducible changes in light intensity. The resulting average force-vector deviation was $0.26 \mathrm{~N}$ (average 3D deviation among the 10 predictions over all measured points). If we only consider applied force intensity (i.e., the force-vector norm) we obtained an average deviation of $0.11 \mathrm{~N}$. A limitation of this experiment is that our force application system itself suffers from declining precision at low force. More precisely, under $1 \mathrm{~N}$, the standard error on the applied force is rapidly $\sim 10 \%$ and the error on applied force can rise to $>50 \%$ (see Supplementary Table S1 for details).

\section{Measurement throughput and computational burden}

An important factor in several robotic applications is the throughput of the system and the computational burden involved. With the imaging system used in our prototype $(1.3 \mathrm{Mpx})$, it would be possible to image simultaneously fibers of $\sim 280$ cells. Using smaller optical fibers or a different magnification it is reasonable to expect to be able to image several thousands of cells with a standard CMOS. In our system, the exposure time was $12 \mathrm{~ms}$, therefore acquisition at $80 \mathrm{~Hz}$ would be feasible. With a more sensitive camera or an improved optical system, much higher acquisition frequencies are possible. Once images are captured, fiber intensity extraction and normalization takes $\sim 80 \mathrm{~ms}$ per frame for 49 cells simultaneously. Force reconstruction from fiber intensity takes on average $28 \mathrm{~ms}$ per cell and frame. This step is the most computationally intensive and might be a current limitation for some applications. These results were obtained on a $2.5 \mathrm{GHz}$ quad-core. As these timings were produced with a poorly optimized MATLAB ${ }^{\circledR}$ implementation, they are meant only to provide an upper bound of the computation burden. In addition to code optimization, closed form solution might exist and results tabulation could be used to achieve orders of magnitude faster computation. Indeed, although precise reconstruction can be 

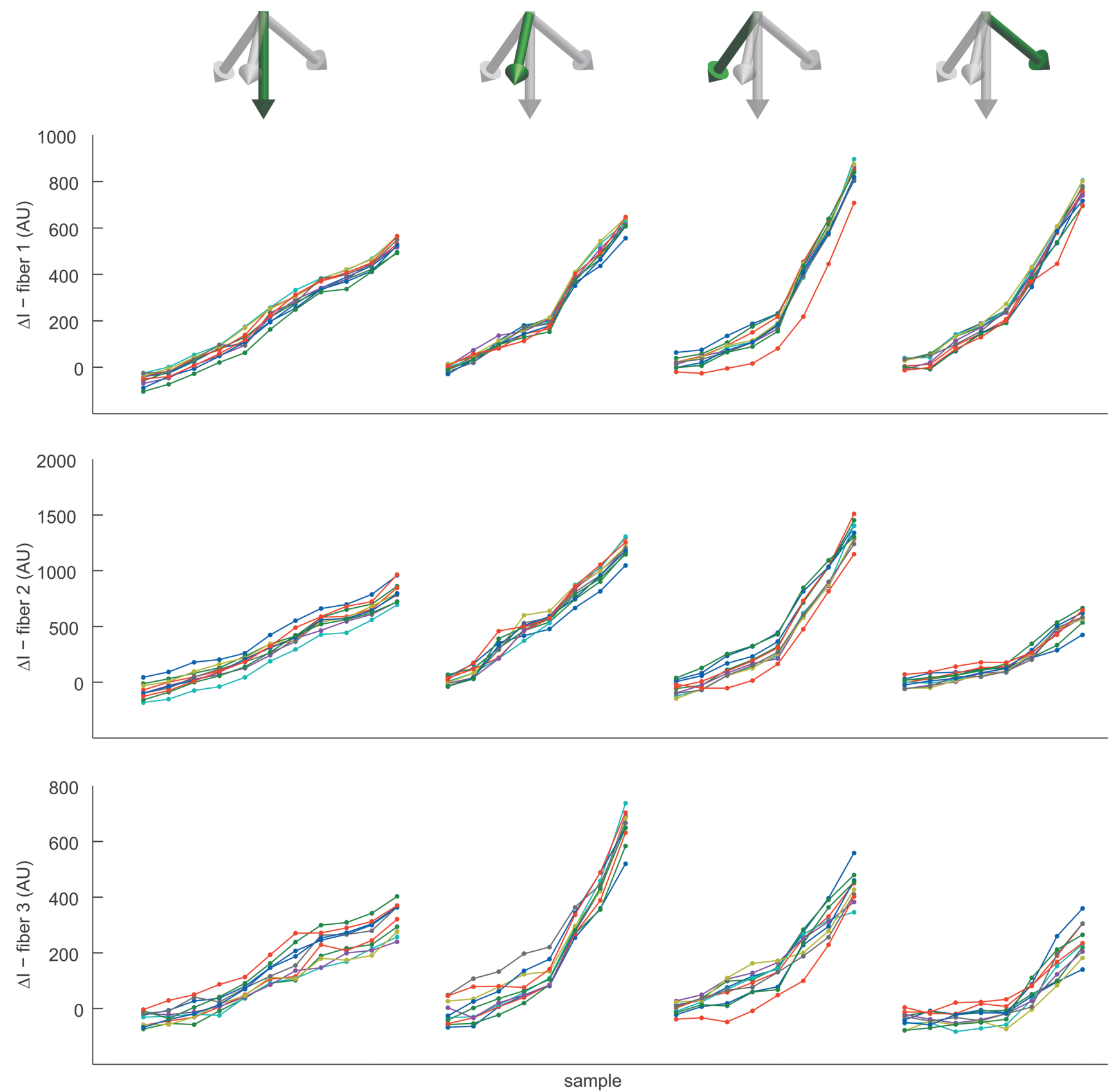

FIG. 5. Experimental reproducibility of force measurements. Four force application sequences with distinct directions were repeated 10 times. Normalized fiber intensities for each fiber of the studied cell are reported here with distinct repetitions arbitrarily color coded. Current variance in intensity across repetitions is expected to come mostly from variations in the actual applied force and from insufficiently compensated injected light variations rather than from sensor noise.

currently computation intensive, determining if a given force threshold has been crossed is extremely fast, allowing the use of this solution also for time-sensitive application such as collision prevention.

\section{Illustrating example}

As a more direct illustration of the regular function of the presented system, we performed a simple experiment as given in Figure 6 and in a Supplementary Video S1. While the carpus remained resting upon the table we applied a pressing force with the index and the auricular fingers alternatively. As we can see by comparing Figure $6 \mathrm{~B}$ and C, the index is capable of stronger force than the auricular, as expected. In addition, looking at tangential components in Figure 6B, we can see that when applying force with the auricular, the movement is less certain, with mild lateral oscillations of the digit. Also, given the respective position of the index and auricular to the point of force application (roughly at the center of the hand), forces were slightly slantwise in 
A
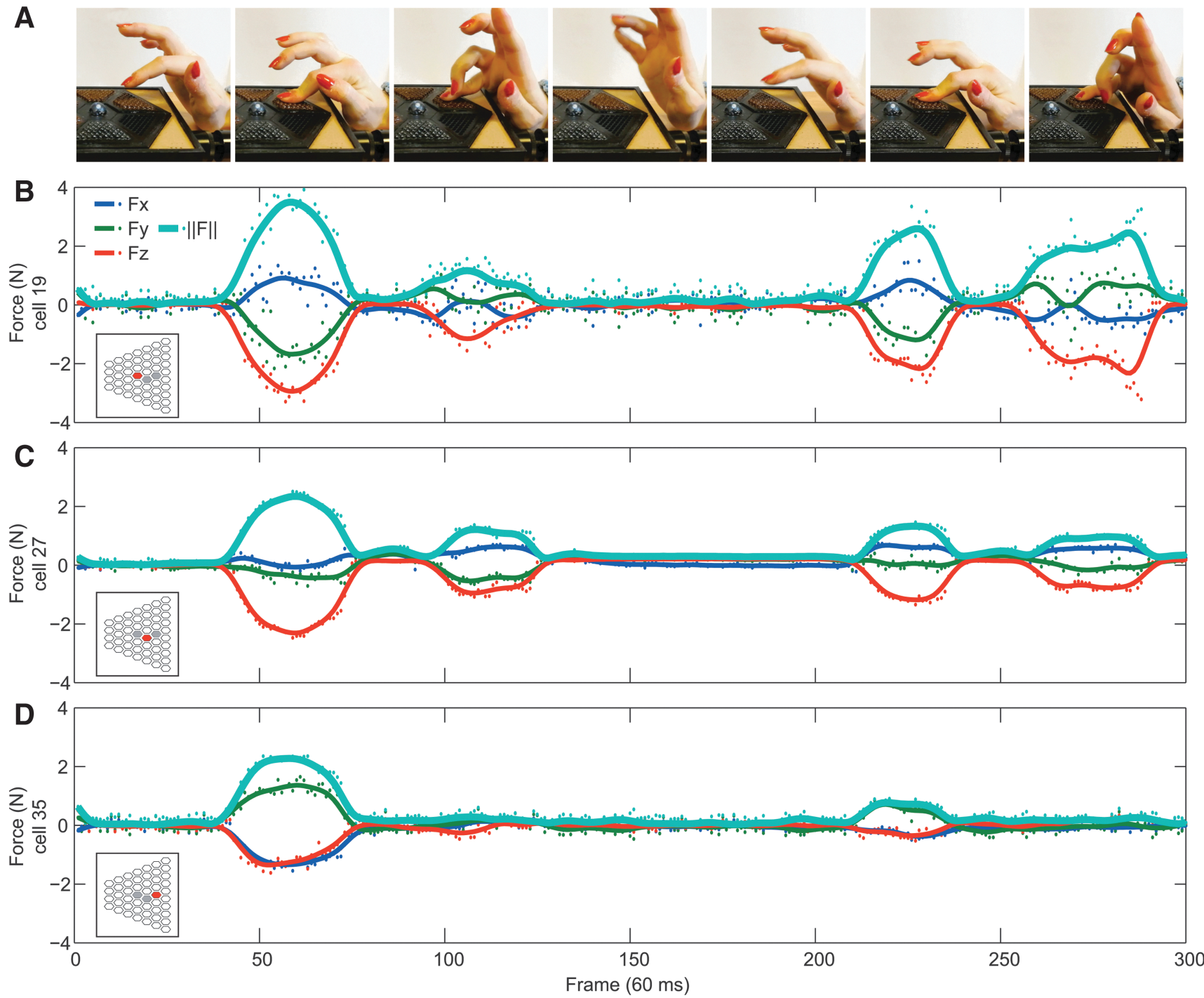

FIG. 6. Force measurement for three different cells from a toy example of experiment where we measure the force exerted by the index and auricular fingers. (A) Snapshots of the experiment sequence, placed approximately at the instants corresponding to the graphs hereunder (see Supplementary Video S1 for details). (B-D) Force measurements time series of cells 19, 27, and 35 (see insert sketch for localization). Full lines are smoothed values from the measurements shown as dots. Three hundred measurements were performed at $60 \mathrm{~ms}$ intervals with a $12 \mathrm{~ms}$ exposure time.

opposite directions for these fingers. Finally, the spatially resolved aspect of our sensor is visible by comparing Figure 6B, C with D: the auricular did not touch the latter cell although it clearly touched its neighbor with nonnegligible force, showing that cells provide mechanically independent measurements.

\section{Conclusion}

We presented a novel force sensor enabling the dynamic measurement of both the direction and intensity of forces in a spatially resolved manner. Modifying simple design parameters, it is possible to tune its key properties. Playing on cell size alters the spatial resolution of measurements and playing on cell stiffness defines the range of measurable force. Because of its modular architecture, the sensing surface can take varied shapes. Using a flat prototype, we demonstrated that it can reconstruct accurately and repeatedly the applied force vector on the target range of $0-2.5 \mathrm{~N}$ in at least a $65^{\circ}$ cone. In its current configuration, this sensor is adapted to the study of small animals or for precision grasps in robotic hands. Forthcoming applications include the use of a cylindrical version of the sensor to measure in situ for the first time, friction forces repartition on hands and feet during arboreal locomotion. This will provide unique insights for climbing biomechanics in primates by revealing the mechanical cues allowing them to move along branches without falling.

Tangential forces also play a key role in grasping complex, deformable, and unknown objects. Mirroring the upcoming characterization of animal hands and feet, it would be informative to equip a robotic hand with our force sensors at its "fingertips" in a similar way as in previous works. $^{15,16}$ This would allow to compare the grasping 
performance when using proprioception only, proprioception and pressure information, or proprioception and 3D force information. This would provide an objective assessment of the impact of the somesthesis degree on the performance of grasping and haptic exploration. In addition, it could motivate the inclusion of this sensor as a component of smart skins.

Besides these prospective research directions, several technical improvements and extensions should be considered. Unlike most current pressure or force sensors, our system is based on an optical measurement of deformations. This engineering direction, which was set aside for nearly two decades, now benefits from game-changing innovations in digital imaging with CMOS, light sources with LEDs, and light guiding with POFs. Of importance, our measurements do not require sophisticated optical hardware such as interferometry-based sensors. We used inexpensive and flexible POFs as light pipes to supply light to sensing elements and back to a central measurement system. This architecture ensures immunity to electromagnetic interference and a low consumption compared with electrical wiring. In addition, the quantity of fibers could be further reduced using wavelength multiplexing. We used a single CMOS sensor to measure the light intensity of each individual fiber for all cells simultaneously. This means that, to a large extent, both the performance (in terms of precision, acquisition speed, or throughput) and cost of this solution depend upon the choice of the imaging system. This ensures great versatility as a same cell sensor array can easily be coupled with either a high-end imaging equipment to provide scientific grade force measurements and a mass market imaging system yielding a miniaturized or cost-effective spatially resolved 3D force sensor. These considerations about image capture also extend to the whole data acquisition and processing chain. Indeed, having image-based measurements, it may be possible to adapt vision-specific hardware and software (e.g., GPUs or FPGA) to improve the postprocessing chain performance in a similar spirit as given in Ascari et al. ${ }^{14}$ Most existing force/ pressure measurement technology requires custom-build electronics, for which cost and performance are driven by capital-intensive R\&D. Each improvement requires additional R\&D and therefore an increase in cost. In contrast, our system is directly connected to standard components and consequently directly benefits from both the economy of scale and the innovation pace of one of the largest and most competitive market in electronics.

Concerning the sensor body, using microfabrication techniques such as soft lithography could enable several promising adaptations upon the presented design. Optical fibers could be replaced by ribbons of soft micrometric light pipes, improving the sensor integration. In addition, it could simplify the assembly process and enable the production of sensors with smaller cells and thus, higher spatial resolution. Finally, it could reduce manufacturing variability, increasing altogether sensor performance.

\section{Acknowledgments}

This work was partially supported by the French PIA IDEFI grant (ANR11-IDFI-0023, Institut Innovant de Formation par la Recherche) and by the SATT Lutech technological transfer company, Paris, France. The authors are grateful to the CR2P laboratory (Centre de Recherche sur la Paléobiodiversité et les Paléoenvironnements, UMR 7207, MNHN, CNRS, UPMC, France) and the CRI (Center for Research and Interdisciplinarity) for granting access to their facilities.

The authors thank Elie Brami for his significant contribution to prototype construction and Hélène Salin, Max Lesaffre, Maxime Huynh, Eric Tordjeman, and Damien Bretegnier for their help and support. Both authors were supported by the FdV (Frontière du Vivant) doctoral school and the University Paris Diderot.

\section{Author Disclosure Statement}

No competing financial interests exist.

\section{Supplementary Material}

Supplementary Table S1

Supplementary Video S1

\section{References}

1. Robles-De-La-Torre G. The importance of the sense of touch in virtual and real environments. IEEE Multimedia 2006;13:24-30.

2. Damasio A, Carvalho GB. The nature of feelings: Evolutionary and neurobiological origins. Nat Rev Neurosci 2013; 14:143-152.

3. Miljkovic-Licina M, Gauchat D, Galliot B. Neuronal evolution: Analysis of regulatory genes in a first-evolved nervous system, the hydra nervous system. Biosystems 2004; 76:75-87.

4. Bartolozzi C, Natale L, Nori F, et al. Robots with a sense of touch. Nat Mater 2016;15:921-925.

5. Lu N, Kim DH. Flexible and stretchable electronics paving the way for soft robotics. Soft Robot 2014;1:53-62.

6. Silvera-Tawil D, Rye D, Velonaki M. Artificial skin and tactile sensing for socially interactive robots: A review. Rob. Auton. Syst 2015;63:230-243.

7. Ascari L, Corradi P, Beccai L, et al. A miniaturized and flexible optoelectronic sensing system for tactile skin. J Micromech Microeng 2007;17:2288-2298.

8. Macefield VG. Physiological characteristics of lowthreshold mechanoreceptors in joints, muscle and skin in human subjects. Clin Exp Pharmacol Physiol 2005;32:135144.

9. Park J, Lee Y, Hong J, et al. Tactile-direction-sensitive and stretchable electronic skins based on human-skin-inspired interlocked microstructures. ACS Nano 2014;8:1202012029.

10. Kim K, Lee KR, Lee, DS, et al. A silicon-based flexible tactile sensor for ubiquitous robot companion applications. J Phys Conf Ser 2006;34:399-403.

11. Noda K, Onoe H, Iwase E, et al. Flexible tactile sensor for shear stress measurement using transferred sub- $\mu \mathrm{m}$ thick Si piezoresistive cantilevers. J Micromech Microeng 2012;22:115025.

12. Tar Á, Cserey G. Development of a low cost 3D optical compliant tactile force sensor (2011 IEEE/ASME International Conference on Advanced Intelligent Mechatronics, AIM). Budapest, Hungary: IEEE, 2011, pp. 236-240.

13. Núñez CG, Navaraj WT, Polat EO, et al. Energyautonomous, flexible, and transparent tactile skin. Adv Funct Mater 2017;27. 
14. Ascari L, Bertocchi U, Corradi P, et al. Bio-inspired grasp control in a robotic hand with massive sensorial input. Biol Cybern 2009;100:109-128.

15. Zaidi L, Corrales JA, Bouzgarrou BC, et al. Model-based strategy for grasping 3D deformable objects using a multi-fingered robotic hand. Rob Auton Syst 2017;95: 196-206.

16. Kaboli M, Yao K, Cheng G. Tactile-based manipulation of deformable objects with dynamic center of mass (2016 IEEE-RAS International Conference on Humanoid Robot). Cancun, Mexico: IEEE, 2016, pp. 752-757.

17. Robles-De-La-Torre G, Hayward V. Force can overcome object geometry in the perception of shape through active touch. Nature 2001;412:445-448.

18. Johnston ID, McCluskey DK, Tan CKL, et al. Mechanical characterization of bulk Sylgard 184 for microfluidics and microengineering. J Micromech Microeng 2014;24: 35017.

19. Wang Z. Polydimethylsiloxane mechanical properties measured by macroscopic compression and nanoindentation techniques. 2011. https://scholarcommons.usf.edu/etd/3402 (accessed January 31, 2019).

Address correspondence to:

Séverine Toussaint

CR2P-Centre de Recherche en Paléontologie-Paris

8 rue Buffon CP38

75231 Paris cedex 05

France

E-mail: severine.toussaint@cri-paris.org 